\title{
Benchmark experiment with iron slab by Time-of-flight technique at CIAE
}

\author{
Yanyan Ding ${ }^{1}$, Yangbo $\mathrm{Nie}^{1}$, Jie Ren ${ }^{1}$, Xichao Ruan ${ }^{1}$, Qi Zhao ${ }^{1}$, Hanxiong Huang ${ }^{1}$, Jie Bao ${ }^{1}$, Haicheng $\mathrm{Wu}^{1}$ \\ ${ }^{1}$ Key Laboratory of Nuclear Data, China Institute of Atomic Energy, Beijing 102413, China
}

\begin{abstract}
In order to validate the evaluated nuclear data, leakage spectra in the range of 0.8 to $15 \mathrm{MeV}$ from samples were measured by time-of-flight (TOF) technique using a D-T neutron source. An experimental system for benchmark validation of nuclear data with slab samples has been set up at China Institute of Atomic Energy (CIAE). In this study, test samples are iron slabs, of which the thickness are $5 \mathrm{~cm}$, $10 \mathrm{~cm}$ and $15 \mathrm{~cm}$, and the measured angles were chosen to be about $60^{\circ}$ and $120^{\circ}$. By comparing measured leakage spectrum with calculated ones by MCNP-4C code, using the data from the CENDL-3.1, ENDF/BVIII.0, JENDL-4.0 and JEFF-3.3 nuclear data files, and the comparison was made by the spectrum shape and by the $\mathrm{C} / \mathrm{E}$ values in different energy regions.
\end{abstract}

\section{Introduction}

The benchmark experimental study on the fusion neutronics plays an important role for validating the accuracy of the evaluated nuclear data, especially the elements that are of interests in nuclear devices, fission and fusion reactors for technologies. Fe is one of such elements that can be used as structural material of nuclear device[1]. A series of experiments on iron[2-5] have been performed to provide the experimental data base for qualifying and validating the iron nuclear data, by using the D-T neutron source or the ${ }^{252} \mathrm{Cf}$ neutron source. However, some discrepancies between measured leakage neutron spectra and MCNP calculated ones with the different evaluated nuclear data libraries have been observed[6].

In order to validate currently available nuclear data files for iron, the leakage neutron spectra from iron slabs were measured and calculated at CIAE by using the integral benchmark facility, which has been successfully used in a series of benchmark experiments [7-10]. The leakage neutron spectra from iron slabs were measured between $0.8 \mathrm{MeV}$ and $15 \mathrm{MeV}$ at emission angles of $60^{\circ}$ and $120^{\circ}$ using TOF method with a BC501A scintillation detector. The results were compared with the calculations using a Monte Carlo code MCNP-4C[11] using the evaluated nuclear data derived from the libraries of CENDL-3.1[12], ENDF/B-VIII.0[13], JENDL-4.0[14] and JEFF-3.3[15]. The comparison was made in both the leakage neutron spectrum and the calculation-to-experiment $\mathrm{ratio}(\mathrm{C} / \mathrm{E})$ of the spectrum integrated over four regions. Description of the experimental arrangement, the measurements, the simulations and the results will be given in the following sections.

\section{Experimental procedure}

The experiment was performed at CIAE, an overview of the experimental arrangement is given in Fig.1.

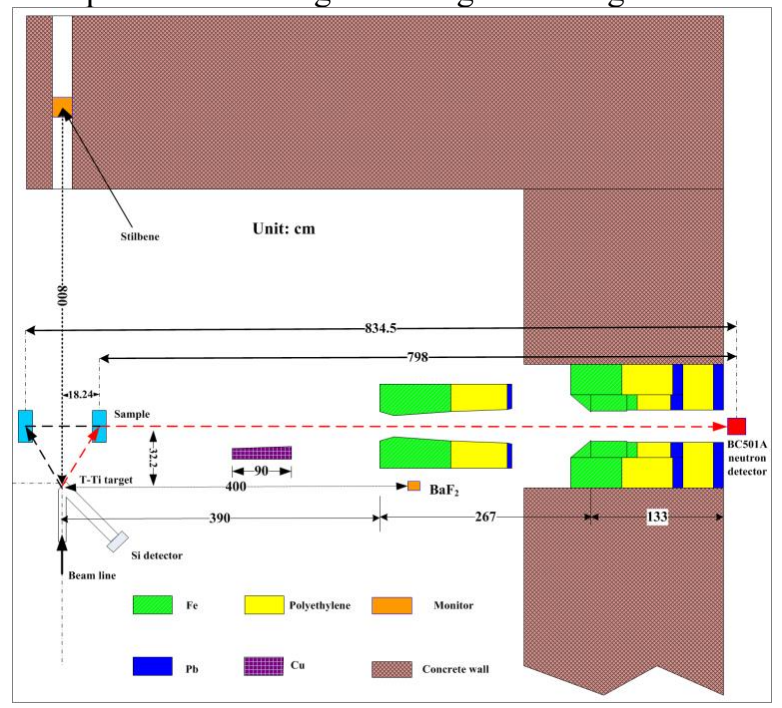

Fig. 1. Experimental arrangement for measuring the neutron leakage spectra from iron slabs.(The red dotted lines indicate the typical neutron paths)

\subsection{Neutron source}

An intense Deuterium-Tritium neutron source facility was used to generate neutrons. The deuteron beam which pulsed and bunched to about 2 ns width (full width at half maximum) was bombarded the tritium target to generate $14.5 \mathrm{MeV}$ neutrons by the $\mathrm{T}(\mathrm{D}, \mathrm{n})^{4} \mathrm{He}$ reactions and the repetition rate was $1.5 \mathrm{MHz}$. The number of source neutrons was estimated by alpha-particle counts 
produced simultaneously in the $T(D, n)^{4} \mathrm{He}$ reactions. The average neutron yield was about $1 \times 10^{9} \mathrm{n} / \mathrm{s}$.

\subsection{Iron slabs}

The cylindrical plates with $13 \mathrm{~cm}$ in diameter and slab plates with surface area of $30 \mathrm{~cm} \times 30 \mathrm{~cm}$ were used in the present experiment, the thicknesses were chosen to be $5 \mathrm{~cm}, 10 \mathrm{~cm}$ and $15 \mathrm{~cm}$, corresponding to $1.08,2.16$ and 3.24 mean free paths for $14.5 \mathrm{MeV}$ neutrons, respectively. The chemical composition of the slabs was $99.9 \%$ iron with small amounts of impurities as shown in Table.1. The density was $7.83 \mathrm{~g} / \mathrm{cm}^{3}$.

Table 1. The compositions of the sample(in weight).

\begin{tabular}{|c|c|}
\hline Nuclide & Mass ratio \\
\hline $\mathrm{Fe}$ & $99.900 \%$ \\
\hline $\mathrm{Si}$ & $0.030 \%$ \\
\hline $\mathrm{C}$ & $0.015 \%$ \\
\hline $\mathrm{Mn}$ & $0.020 \%$ \\
\hline $\mathrm{Al}$ & $0.020 \%$ \\
\hline $\mathrm{Pb}+\mathrm{Sn}+\mathrm{Bi}+\mathrm{Cu}+\mathrm{S}+\mathrm{P}$ & $0.015 \%$ \\
\hline
\end{tabular}

\subsection{The detector and collimator}

The neutron spectra leaking from slabs were detected by a BC501A liquid scintillation detector, which was located behind the concrete wall at about $8.15 \mathrm{~m}$ flight path in a direction perpendicular to the $\mathrm{D}+$ beam line.

A stilbene scintillation crystal of $5.08 \mathrm{~cm}$ in diameter was placed at about $8 \mathrm{~m}$ from the T-Ti target along with the $\mathrm{D}+$ beam line for monitoring the neutron pulse shape spectra. Another $\mathrm{BaF}_{2}$ scintillation crystal, which was located at about $4 \mathrm{~m}$ from the T-Ti target in a direction perpendicular to the $\mathrm{D}+$ beam, was used to match the stilbene scintillation crystal to monitor the neutron source pulse shape.

In front of the neutron detector, a collimator, which was embedded inside the concrete wall of $200 \mathrm{~cm}$ thick with a hole of $10 \mathrm{~cm}$ diameter, was set to shield the neutron detector from background neutrons. A precollimator, which was made of iron, polyethylene and lead, was also placed between the sample and the detector to reduce the neutron background. Another 90 $\mathrm{cm}$ long shadow bar of $\mathrm{Cu}$ was also installed to eliminate the direct fast neutrons from the T-Ti source. Using such a heavy shielding and collimating system, a high foreground/background ratio has been achieved[16].

\subsection{Measuring procedure and data processing}

Even though the background neutrons were significantly suppressed by collimator, a background correction was necessary in the measured spectra. In order to make the background correction, two runs of measurement with sample in (foreground) and sample out (background) were performed for each sample and angle which was shown in Fig.2.

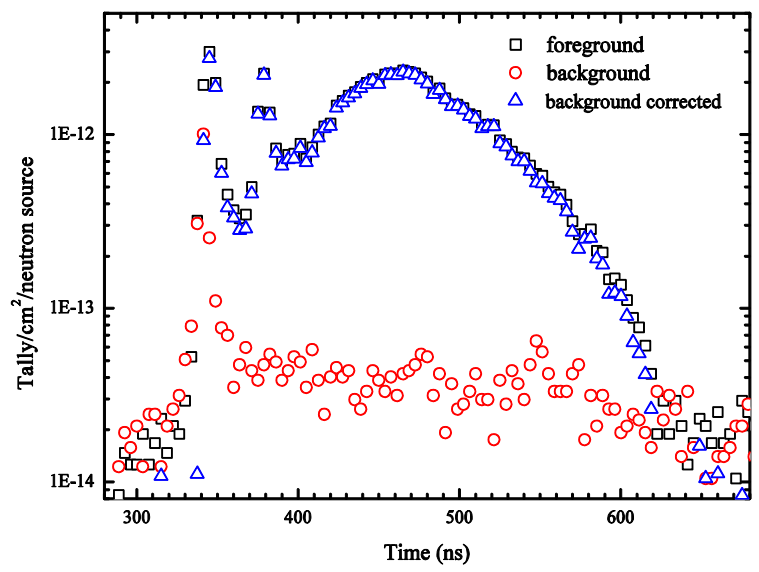

Fig.2 Experimental neutron leakage time spectra measured at $60^{\circ}$.

The measured angles were chosen to be nearly $60^{\circ}$ and $120^{\circ}$, transformed by changing the target (sample) position along the leakage neutron direction, which results in change of the neutron emission angle from the Tritium-Titanium (T-Ti) target. As shown in Fig. 1, we put the sample on the right side of the dash line if measuring the leakage spectrum at $60^{\circ}$, and moved to the left side while measuring at $120^{\circ}$.

Polyethylene slab samples $(\Phi 13 \mathrm{~cm} \times 6 \mathrm{~cm}$ and 30 $\mathrm{cm} \times 30 \mathrm{~cm} \times 6 \mathrm{~cm}$ ) were used as the standard sample to calibrate the absolute neutron yield from iron samples. The leakage neutron spectrum from the polyethylene sample was measured at $60^{\circ}$. The comparison of the measured spectrum and the corresponding one calculated by MCNP-4C with the ENDF/B-VIII.0 evaluated nuclear data can be found in Fig.3 and Fig.4, from which it can be observed that the experimental result is well reproduced by the calculated one in the whole neutron energy range. This result indicates that the experimental apparatus and the data analysis procedures work well.

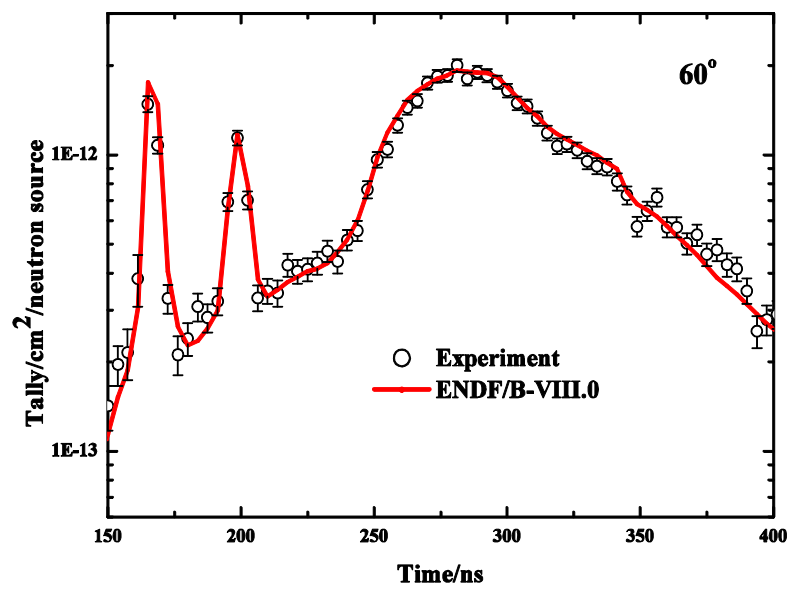

Fig.3 Leakage neutron spectrum from polyethylene sample at $60^{\circ}(\Phi 13 \mathrm{~cm} \times 6 \mathrm{~cm})$ 


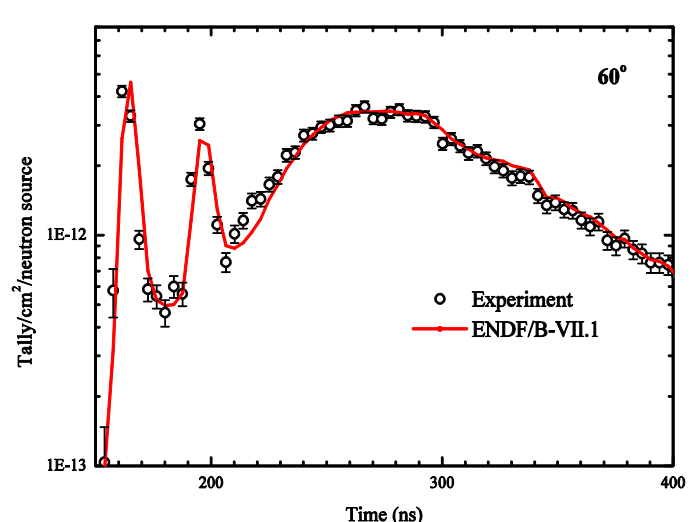

Fig.4 Leakage neutron spectrum from polyethylene sample at $60^{\circ}(30 \mathrm{~cm} \times 30 \mathrm{~cm} \times 6 \mathrm{~cm})$.

The uncertainties of the present experiment mainly come from the statistical and the systematic ones. The statistical uncertainty is about $5 \%$, which includes the uncertainties of the neutrons number in per time bin $(\sim 5 \%)$, the number of $n-p$ scatting neutrons from experiment $(<0.5 \%)$ and MCNP calculation $(<0.5 \%)$, respectively, the number of $\alpha$ particles detected for the polyethylene $(<0.5 \%)$ and iron samples $(<0.5 \%)$. The systematic uncertainty was mainly caused by the relative neutron detection efficiency $(\leq 3 \%)$ and the scattering angle ambiguity $(\leq 1 \%)$. Overall, the total uncertainty is less than $10 \%$.

\section{Monte Carlo simulation}

For comparison of the experimental results with the calculation, the neutron leakage spectra are simulated by the MCNP-4C code using the evaluated data of iron from the CENDL-3.1, ENDF/B-VII.1, JENDL-4.0 and JEFF-3.3 libraries. In the MCNP simulation, the experimental configuration was modeled in detail, and the detailed experimental parameters were taken into account.

For sample with $13 \mathrm{~cm}$ in diameter the calculations for two angles of $60^{\circ}$ and $120^{\circ}$ were performed with the model shown in Fig. 5. Others with sueface area of 30 $\mathrm{cm} \times 30 \mathrm{~cm}$, we use the model shown in Fig. 6. Ring detector estimator $(5.08 \mathrm{~cm}$ in diameter, the same diameter as the BC501A detector) was used to tally the leakage neutron TOF spectra for comparing with the measured ones in both two models. The left sample was filled with air when the simulation was performed for $60^{\circ}$, while the right sample was filled with air for $120^{\circ}$ simulation. For the background simulation, both samples were filled with air. The neutron histories adopted were $10^{9}$ and the statistical uncertainties of each time bin were smaller than $1 \%$.

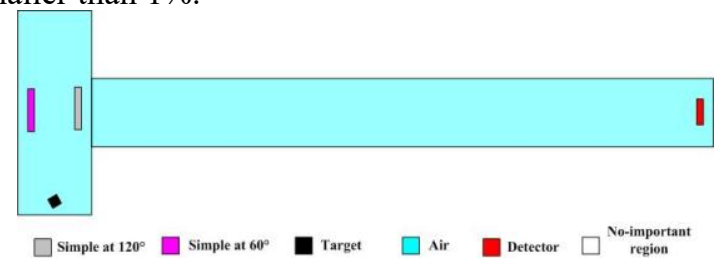

Fig.5 Model for the MCNP calculations with sample surface area of $\Phi 13 \mathrm{~cm}$.

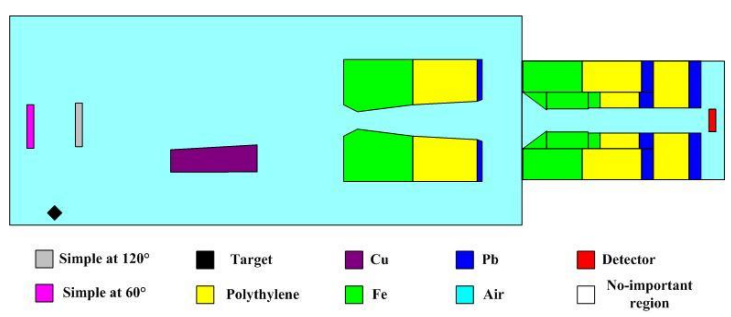

Fig.6 Model for the MCNP calculations with sample surface area of $30 \mathrm{~cm} \times 30 \mathrm{~cm}$.

\section{Results and discussion}

\subsection{Result of $\Phi 13 \mathrm{~cm}$}

The measured leakage neutron spectra for the iron sample with $13 \mathrm{~cm}$ in diameter at $60^{\circ}$ and $120^{\circ}$ are shown in Fig. 7 comparing with the calculated ones using the four evaluated nuclear data libraries. The $\mathrm{C} / \mathrm{E}$ values of the spectra integrated over specified energy ranges are also shown in Fig. 8.
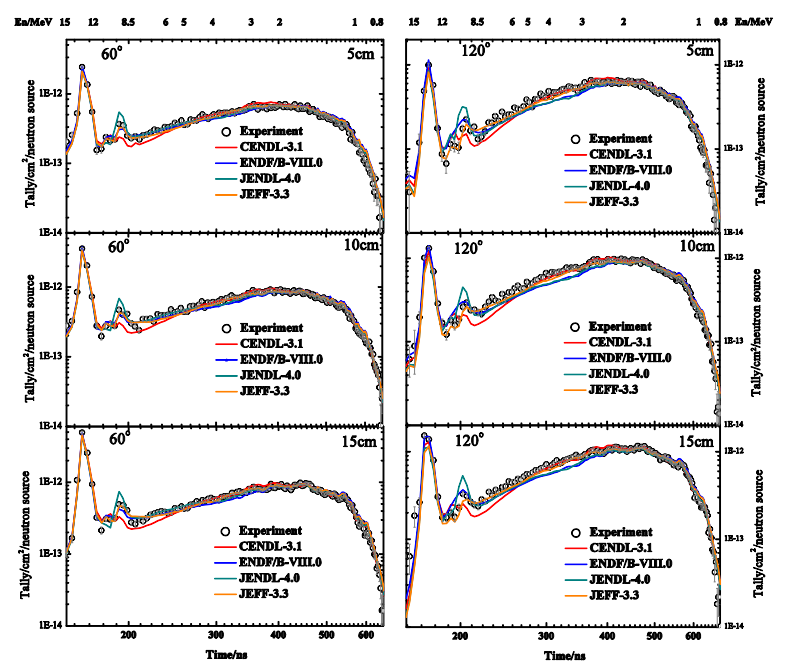

Fig.7 The comparison of measured and calculated leakage neutron spectra from iron sample $(\Phi 13 \mathrm{~cm})$.

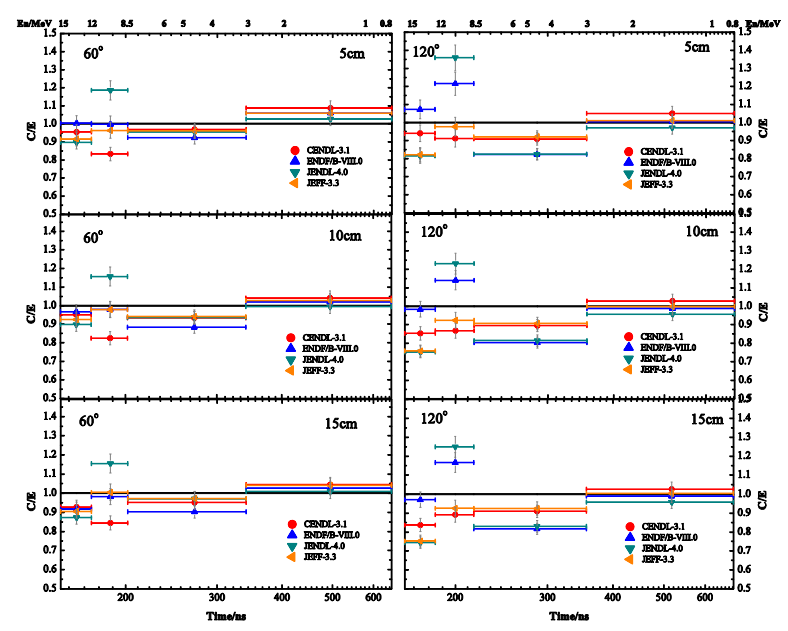

Fig.8 The C/E values integrated over the specified energy ranges at $60^{\circ}(\Phi 13 \mathrm{~cm})$. 


\subsection{Result of $30 \mathrm{~cm} \times 30 \mathrm{~cm}$}

For iron sample with surface area of $30 \mathrm{~cm} \times 30 \mathrm{~cm}$, Fig. 9 shows the measured and calculated neutron leakage spectra at $60^{\circ}$ and $120^{\circ}$. The $\mathrm{C} / \mathrm{E}$ values of the spectra integrated over specified energy ranges are also shown in Fig. 10.

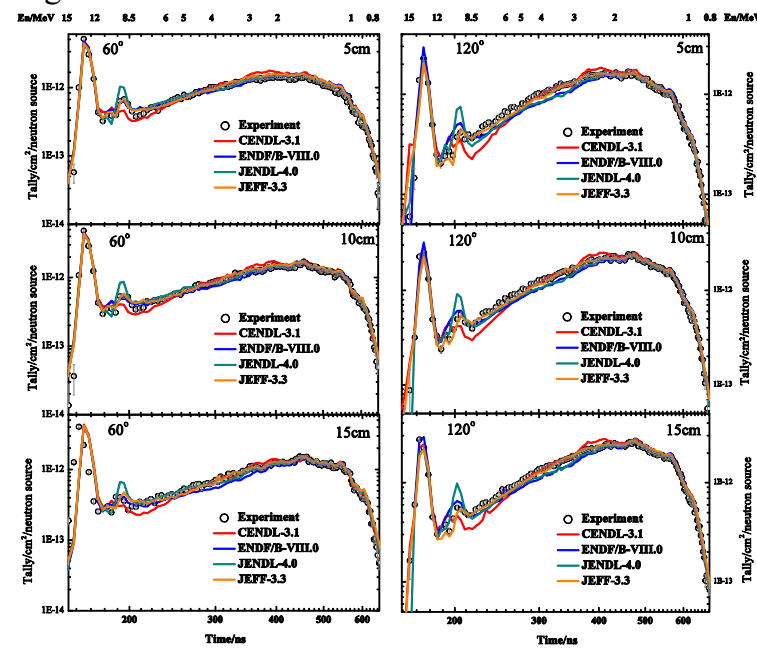

Fig.9 The comparison of measured and calculated leakage neutron spectra from iron sample $(30 \mathrm{~cm} \times 30 \mathrm{~cm})$.

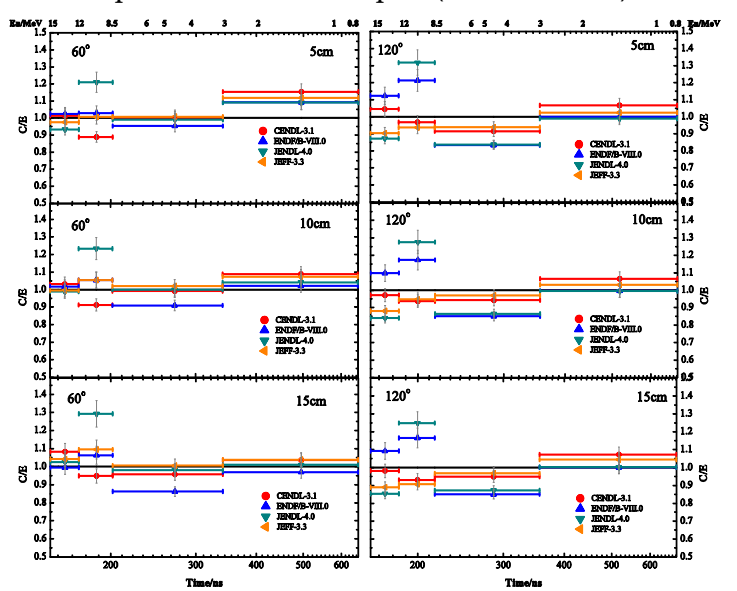

Fig.10 The $\mathrm{C} / \mathrm{E}$ values integrated over the specified energy ranges at $60^{\circ}(30 \mathrm{~cm} \times 30 \mathrm{~cm})$.

From above results, we can conclude that:

(1) In the $12-15 \mathrm{MeV}$ range, most contribution comes from the elastic scattering. The calculated scattering peaks with the ENDF/B-VIII.0 library well reproduce the experimental ones. The calculated neutron spectra with the CENDL-3.1, JENDL-4.0 and JEFF-3.3 libraries are consistent with the experiment at $60^{\circ}$, but underestimated $10 \%-25 \%$ at $120^{\circ}$.

(2) In the medium energy range, $8.5-12 \mathrm{MeV}$, most contribution comes from the discrete inelastic scattering. The calculated neutron spectra with the JENDL-4.0 library are overestimated around $20 \%$ at both $60^{\circ}$ and $120^{\circ}$. At $60^{\circ}$, the results with the CENDL-3.1 library are underestimated around $15 \%$, and at $120^{\circ}$, the results with the ENDF/B-VIII.0 are overestimated by more than $15 \%$.

(3) In the $3-8.5 \mathrm{MeV}$ range, most contribution comes from the continuous level of inelastic scattering. The calculated neutron spectra with the CENDL-3.1 and
JEFF-3.3 libraries agree with the experiment within 11\% at both $60^{\circ}$ and $120^{\circ}$, while the results with the ENDF/B-VIII.0 and JENDL-4.0 libraries are underestimated about $20 \%$ at $120^{\circ}$.

(4) In the $0.8-3 \mathrm{MeV}$ range, the calculated results from all libraries agree with the experimental ones within $10 \%$.

\subsection{Discussion}

In order to find the source of these discrepancies between the calculated results and the experimental ones, the total and several partial cross sections in the evaluated nuclear data libraries are studied. The contributions of different reaction channels to the leakage neutron spectra for iron at incident neutron energy of $14.5 \mathrm{MeV}$ are shown in Fig.11, it can be found that the emission neutrons mainly come from the (n, el), $(\mathrm{n}, \mathrm{inl})$, and $(\mathrm{n}, 2 \mathrm{n})$ reactions, and the $(\mathrm{n}, \mathrm{n} \alpha)$ and $(\mathrm{n}, \mathrm{np})$ show minor contributions.

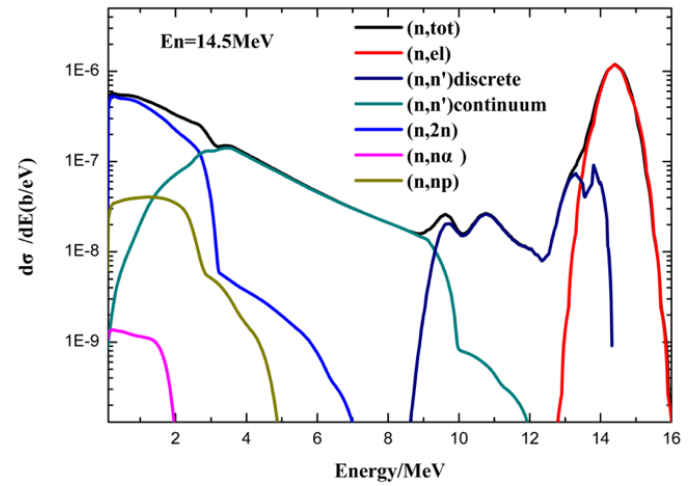

Fig.11 The distributions of leakage neutron spectra from different reactions for ${ }^{\text {nat }} \mathrm{Fe}$ (Retrieved from the CENDL-3.1 library) .

The angular distributions of the neutron elastic scattering for iron are plotted in Fig.12 at the incident neutron energy of $14.5 \mathrm{MeV}$, the cross sections from the ENDF/B-VIII.0 are much closer to most known experimental data than those from other libraries, especially at $60^{\circ}$, while the cross sections from the JENDL-4.0 and JEFF-3.3 libraries are lower than the ENDF/B-VIII.0 ones slightly at $60^{\circ}$ and significantly at $120^{\circ}$, which causes the differences in the elastic scattering peak of the neutron spectra.

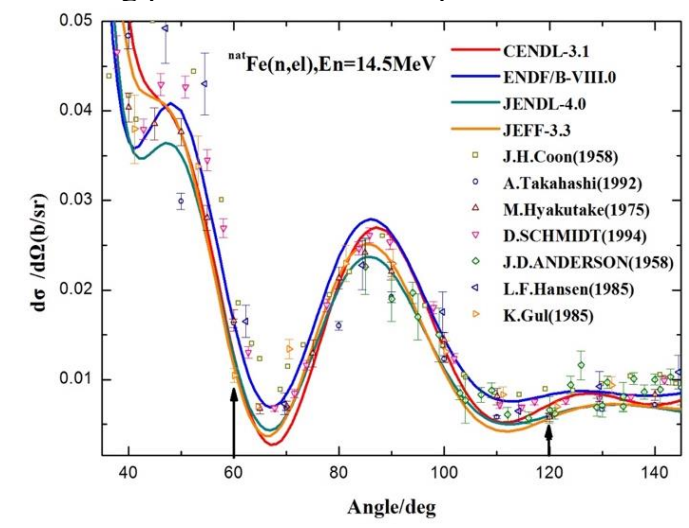

Fig.12 The angular distributions of elastic cross section for iron from different evaluated files. 
The contributions from the continuum inelastic scattering at incident neutron energy of $14.5 \mathrm{MeV}$ are shown in Fig.13, a small, but clear peak is observed at the $8.5-11 \mathrm{MeV}$ range in the spectrum retrieved from the JENDL-4.0, which causes the discrepancies between the measured spectra and the calculated ones at this energy range.

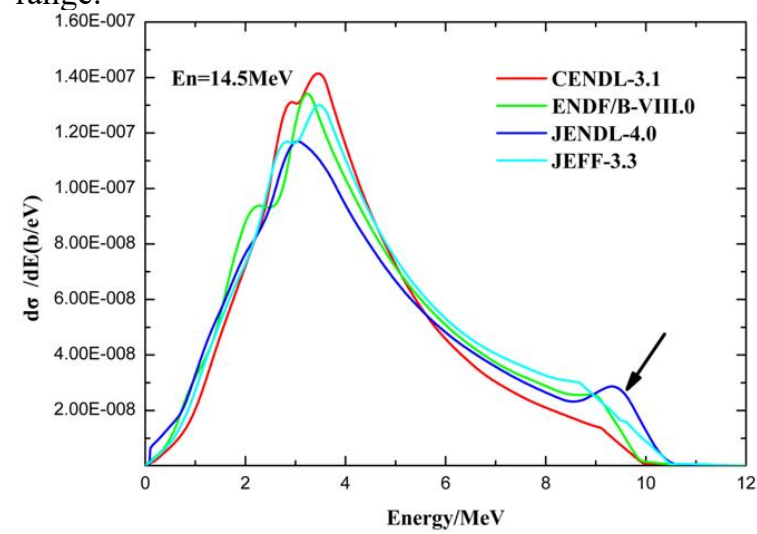

Fig.13 The contributions from the continuum inelastic scattering for iron at the incident neutron energy of $14.5 \mathrm{MeV}$

\section{Summary}

In this work, the validations of the evaluated nuclear data for iron were performed with two kind of samples $(\Phi 13$ $\mathrm{cm}, 30 \mathrm{~cm} \times 30 \mathrm{~cm}$ ), the leakage neutron spectra measured by time-of-flight method at $60^{\circ}$ and $120^{\circ}$. The measured neutron energy range was $0.8-15 \mathrm{MeV}$. The theoretical calculations were carried out by MCNP-4C Monte Carlo code using the evaluated nuclear data of the CENDL-3.1, ENDF/B-VIII.0, JENDL-4.0 and JEFF-3.3 libraries. From the comparisons, it can be observed that in general the calculated neutron spectra with the CENDL-3.1 and JEFF-3.3 show better agreement with the measured ones than those with other libraries. The calculated neutron spectra with the CENDL-3.1 are underestimated slightly in the $8.5-13 \mathrm{MeV}$ energy range; the calculated results with the ENDF/B-VIII.0 are overestimated around $15 \%$ in the $8.5-13 \mathrm{MeV}$ range but underestimated about $20 \%$ in the $3-8.5 \mathrm{MeV}$ range at $120^{\circ}$; the calculated results with the JENDL-4.0 are significantly overestimated in the $8.5-13 \mathrm{MeV}$ range; and the calculated results with the JENDL- 4.0 and JEFF- 3.3 are underestimated around $20 \%$ in the elastic scattering peak at $120^{\circ}$. These discrepancies originate from the improper evaluation of the angular distributions of the neutron elastic scattering and the secondary neutron energy distribution. The data in evaluated nuclear data libraries need to be improved as required in different energy ranges.

\section{Acknowledgements}

This work was supported by national nature science foundation of China (No. 11790320, 11790321, 11790323), the continuous Basic Scientific Research Project (No. WDJC-2019-09).

\section{References}

1. CHADWICK M B, DUPONT E, BAUGE E, et al. Nuclear Data Sheets. 118 (2014) 1.

2. Y. Oyama, K. Kosako, H. Maekawa, et al. Nucl. Sci. Eng. 115 (1993) 24.

3. L. F. Hansen, J. D. Anderson, P. S. Brown, et al. Nucl. Sci. Eng. 51 (1973) 278.

4. P. Bem, U. Fischer, S. Simakov, et al. Fusion Engineering and Design 69 (2003) 479.

5. B. Jansky, E. Novak, Z. Turzik, et al. Nucl. Instr. Methods Phys. Res. A 476 (2002) 358.

6. WENNER M, HAGHIGHAT A, ADAMS J M, et al. Nucl. Sci. Eng. 170 (2012) 207.

7. Yangbo Nie, Jie Bao et al. Benchmarking of evaluated nuclear data for uranium by a $14.8 \mathrm{MeV}$ neutron leakage spectra experiment with slab sample[J]. Annals of Nuclear Energy, 37(2010):1456-1460.

8. R.Han, R Wada et al. Fast neutron scattering on Gallium target at 14.8 MeV[J]. Nuclear Physics A, 936(2015):17-28.

9. Y.Nie, J.Ren et al. The benchmark experiment on slab beryllium with D-T neutrons for validation of evaluated nuclear data[J]. Fusion Engineering and Design, 105(2016):8-14.

10. Zuokang Lin, Yangbo Nie et al. Benchmarking of 232Th evaluation by a $14.8 \mathrm{MeV}$ neutron leakage spectra experiment with slab samples[J]. Annals of Nuclear Energy, 96(2016):181-186.

11. Briesmeister. J, 2000. MCNP-A general Monte Carlo N-particle transport code system, Version 4C, Report LA 13709-M(2000) Los Alamos.

12. Z.G. Ge, Z.X. Zhao, H.H. Xia, et al. J. Korean Phys. Soc. 59 (2011) 1052.

13. D. A. Brown, M. B. Chadwick, R. Capote, et al. Nuclear Data Sheets. 148 (2018) 1.

14. K. Shibata, O. Iwamoto, T. Nakagawa et al. J. Nucl. Sci. Technol. 48 (2011) 1.

15. www.oecd-nea.org/dbdata/jeff/jeff33

16. Xinggang Cai, Yangbo Nie et al. Design of a precollomator system for neutronics benchmark experiment[J]. Nuclear Techniques, 2013,(01):8-13.

17. Y. Nie, J. Ren, X. Ruan, et al. Fusion Engineering and Design 105 (2016) 8. 